\title{
Reconsidering Mathematics Education for the Future
}

\author{
Koeno Gravemeijer, Fou-Lai Lin, Michelle Stephan, Cyril Julie \\ and Minoru Ohtani
}

There is a broad consensus that we should foster 21 st century skills. Mathematics education seems a perfect place to work on those skills, yet, which of the competencies that are assembled under the label 21 st century skills can, and should, be fostered is still an open question. In addition to introducing 21 st century skills in the school curriculum, changes will have to be made in content goals. This is especially the case for mathematics education. Increasingly, machines are doing all kinds of mathematical calculations; at the same time mathematics becomes invisible while disappearing in black boxes. Consequently, it will become important to understand the mathematics in those black boxes, and to know how to work with it. This touches on the content of mathematics education, which has to be adapted accordingly. Much research has already been done on getting a handle on mathematics at today's workplace - which proves to be idiosyncratic and interwoven with tools and practices. Those elements will of course stay relevant for the times to come. If we look ahead, however, more fare-reaching issues emerge. We will have to attune mathematics education to the fact that computers and computerized machines do most mathematical work outside school.

In order to begin a conversation on what mathematics education looks like in the future, the Working Group team assembled a variety of research articles on this issue and invited interested ICMI participants to read them prior to attending the Working Sessions. We intended for these articles to provide a frame for discussing what mathematics content and processes will be relevant in our society in 20 years.

In the first session, Gravemeijer presented a short talk to orient participants to the aims of the sessions: How can mathematics education prepare students for meaningful participation in the future, digital society? Participants then met in self-formed groups to brainstorm answers to the question: What mathematics will citizens need, and what is the role of general abilities known as 21 st century skills?

\footnotetext{
K. Gravemeijer $(\bowtie)$

Eindhoven University of Technology, Eindhoven, The Netherlands e-mail: koeno@gravemeijer.nl

(C) The Author(s) 2017

G. Kaiser (ed.), Proceedings of the 13th International Congress on Mathematical

Education, ICME-13 Monographs, DOI 10.1007/978-3-319-62597-3_93
} 
Afterwards, participants shared out and together created an inventory of topics for consideration: Statistics and probability, variable and function, 3D geometry, measurement, basic mathematics/some advanced, interpreting results of data, quantitative literacy, visualization, critical thinking, problem solving and posing, modeling, deduction/logic/proof, argumentation and communication, collaboration, representation, financial literacy and digital mathematics.

In the second session, the team displayed the Inventory of Topics and asked participants to choose an area to develop more thoroughly with a small group. Results of these discussions yielded several implications: (1) it will be important to know the meaning of basic operations, and to work with number systems, properties and relations; (2) three-dimensional geometry should be emphasized, especially visualization and the relation between the digital world and geometry; (3) modeling processes need to have greater priority, and should involve more open problems; (4) data analysis processes - using technology — are critical, especially in contexts from other disciplines; including thinking backwards when interpreting results; (5) special attention will have to be given to mathematical argumentation, and communication.

Open Access Except where otherwise noted, this chapter is licensed under a Creative Commons Attribution 4.0 International License. To view a copy of this license, visit http://creativecommons. org/licenses/by/4.0/.

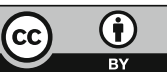

